\title{
Gastric Cancer of "Crawling Type" Detected by Additional Gastrectomy After Endoscopic Submucosal Resection
}

\author{
YASUHIRO HARUTA ${ }^{1}$, RYOTA NAKANISHI ${ }^{1}$, TOMOKO JOGO ${ }^{1}$, YUICHIRO NAKASHIMA ${ }^{1}$, \\ HIROSHI SAEKI ${ }^{1}$, EIJI OKI ${ }^{1}$, MINAKO FUJIWARA ${ }^{2}$, YOSHINAO ODA ${ }^{2}$ and YOSHIHIKO MAEHARA ${ }^{1}$ \\ ${ }^{1}$ Department of Surgery and Science, Graduate School of Medical Sciences, \\ Kyushu University, Fukuoka, Japan; \\ ${ }^{2}$ Department of Anatomic Pathology, Pathological Science, \\ Graduate School of Medical Sciences, Kyushu University, Fukuoka, Japan
}

\begin{abstract}
Crawling type" gastric cancer (GC) is known as a rare variant of early GCs, which is difficult to diagnose at an early stage because of low-grade nuclear atypia and a morphology mimicking intestinal metaplasia. This is a case report of a 69-year-old woman who was diagnosed with early-stage gastric cancer. She had endoscopic submucosal resection (ESD) and histologically, both horizontal and vertical margins were negative. Seven months after ESD, a new lesion of the stomach was detected by follow-up gastroscopy. Laparoscopic distal gastrectomy was performed and "crawling type" glands were observed throughout the whole area of the tumor. We should keep this variant in mind, especially when a tumor is superficial depressed or superficial flat type in the middle of the stomach. Careful observation with multiple biopsies of all mucosal layer and a re-biopsy is the key procedure for obtaining the right diagnosis. Endoscopic and histological characteristics should also be reviewed.
\end{abstract}

Endoscopic treatment for early gastric cancer (GC) has been widespread. The indication for endoscopic resection is restricted to tumors such as intramucosal lesions, smaller than $2.0 \mathrm{~cm}$ which are clinically diagnosed as having no lymph node metastasis, according to the National Comprehensive Cancer Network (NCCN) guidelines. "Crawling type" GC is known as a rare variant of early $\mathrm{GC}$, which accounts for 2-3\% of early GCs $(1,2)$. Endoscopically, the tumor is ill-defined, with a flat appearance and indistinct borders due to the lack of

Correspondence to: Dr. Yasuhiro Haruta, Department of Surgery and Science, Graduate School of Medical Sciences, Kyushu University, 31-1, Maidashi, Higashi-ku, Fukuoka 812-8582, Japan. Tel: +81 926425466, Fax: +81 926425482, e-mail: haruta_y@surg2.med.kyushuu.ac.jp

Key Words: Early gastric cancer, Crawling type, WHY lesion. contrast against the background mucosa (3). This variant is also characterized by low-grade nuclear atypia and a morphology mimicking intestinal metaplasia. Therefore, it is difficult to be diagnosed in the early stages of disease, and the endoscopic resection often ends up with high rates of incomplete resection and positive lateral margins (4). We experienced a case of "crawling type" GC, which was difficult to diagnose by endoscopic and pathological investigations before and after ESD, and was eventually detected after an additional gastrectomy. We also reviewed the endoscopic and histological clues in order to decide the appropriate approach for this type of GC.

\section{Case Report}

A 69-year-old woman was referred to our hospital with nausea and epigastralgia. A gastric tumor was found by upper gastroscopy. Endoscopically, it was a shallow depressed lesion at the lesser curvature of the middle body of the stomach (Figure 1a, 1b). Narrow band imaging (NBI) technique showed the relatively clear demarcation line, and that the micro surface pattern was irregular or absent, and micro vascular pattern was irregular. The first biopsy revealed well to moderately differentiated adenocarcinoma. $\mathrm{X}$-ray fluoroscopic examination also showed a slightly depressed lesion at the same site (Figure 1c), and computed tomography (CT) revealed that it had no concurrent lymph node and distant metastasis. Endoscopic submucosal resection (ESD) was performed under the diagnosis of early GC (cT1a, N0, M0, cStageIA). Histological examination revealed a well to moderately differentiated adenocarcinoma, $23 \times 22 \mathrm{~mm}$ in size which was restricted to the mucosa with no vessel and lymphatic invasions. Both horizontal and vertical margins were negative (pT1a, ly-, v-, UL-, pHM0, pVM0) (Figure 1d). Consistent with the criteria of curative resection, the patient was followed up every 3 months without any additional treatment. 


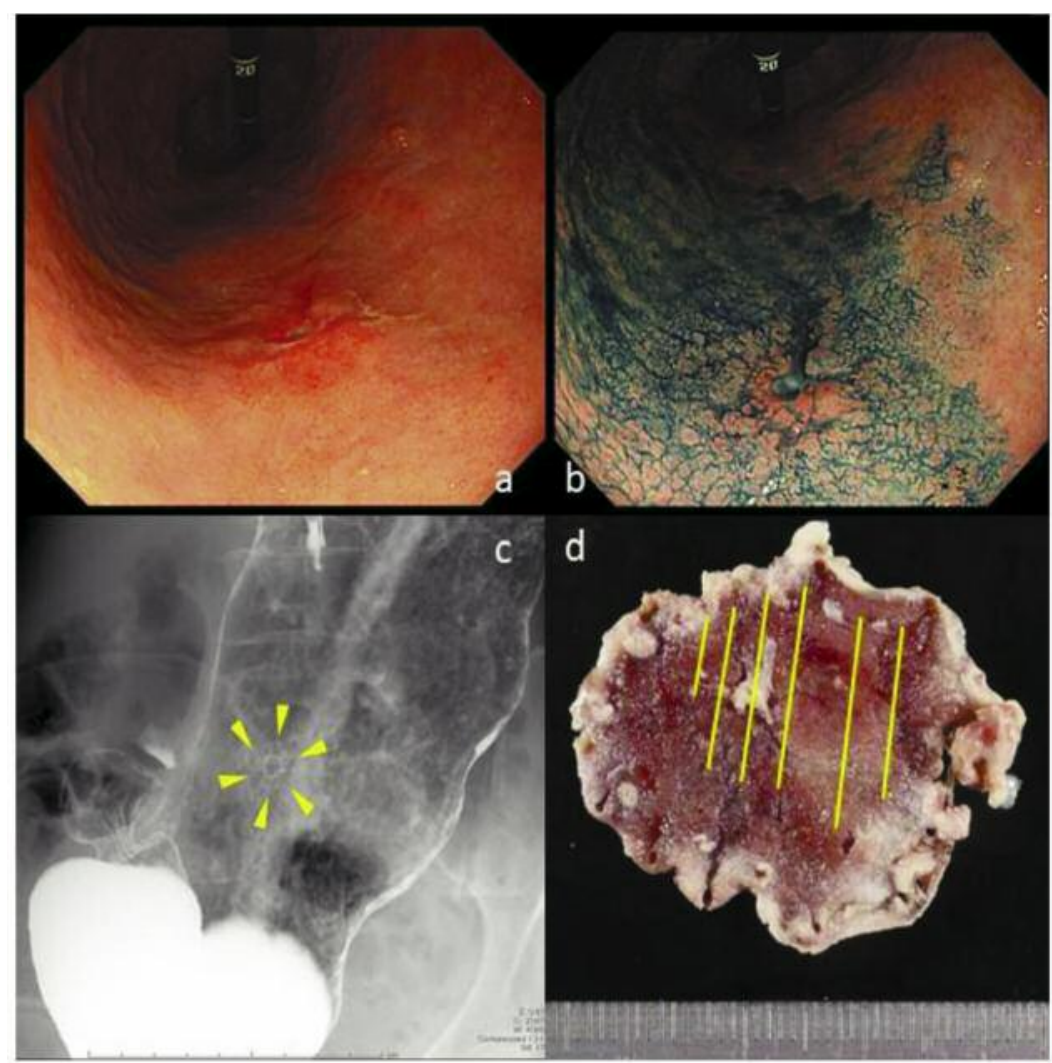

Figure 1. The macroscopic findings of the tumor before and after endoscopic resection. a) Endoscopically, the tumor was detected as a shallow depressed lesion at the lesser curvature. $b$ ) The borders were relatively well defined by the indigocarmine staining. $c$ ) X-ray fluoroscopic examination also showed a shallow depressed lesion. d) Histologically, the resection margins of the specimen were free of carcinoma cells. Yellow line showed the gastric cancer.

Seven months after ESD, an irregular change of the ESD scar and a new low elevated lesion at the posterior wall in the lower body of the stomach were detected by follow-up gastroscopy (Figure 2a, 2b). The mucosa between the two lesions was normal. The biopsies of these lesions revealed a well differentiated adenocarcinoma at the scar and moderately and poorly differentiated adenocarcinoma at the low elevated lesion. CT did not reveal any concurrent lymph node or distant metastasis. Laparoscopic distal gastrectomy was performed under the diagnosis of recurrent gastric cancer after ESD resection. The proximal resection line was determined by the preoperative clipping at the negative biopsy site. Histological examination revealed that the tumor was a well to poorly differentiated adenocarcinoma with lymphoid invasion but with no venous invasion. The size of the tumor was $175 \times 90 \mathrm{~mm}$ and it involved the ESD scar and the low elevated lesion (Figure 3a). The resection margins were negative, but were close to the proximal resection margin (ly2, v0, pPM0 (2 mm), pDM0 (66 mm), pR0). Poorly differentiated adenocarcinoma cells were detected only in the submucosa, and were restricted at the low elevated lesion (Figure 3b). "Crawling type" glands were observed throughout the whole area of the tumor, which spread laterally in the lamina propria but not in the gastric glands, (Figure 3a, 3c). There was no lymph node metastasis. Because the proximal resection margin was only $2 \mathrm{~mm}$, we performed the biopsies around the anastomosis site every 4 months in addition to the CT performed every 6 months. 13 months after the gastrectomy, there was no sign of recurrence.

\section{Discussion}

"Crawling type" GC is characterized by low grade nuclear atypia and morphology mimicking intestinal metaplasia with a laterally spreading pattern (2). "Crawling type" glands are known as one of the characteristics of extremely welldifferentiated adenocarcinomas (EWDA) of the stomach. (4). It is reported that EWDA accounts for less than $0.2 \%$ of all GCs and $1.9-2.9 \%$ of early GCs $(1,5,6)$. Endoscopically, 


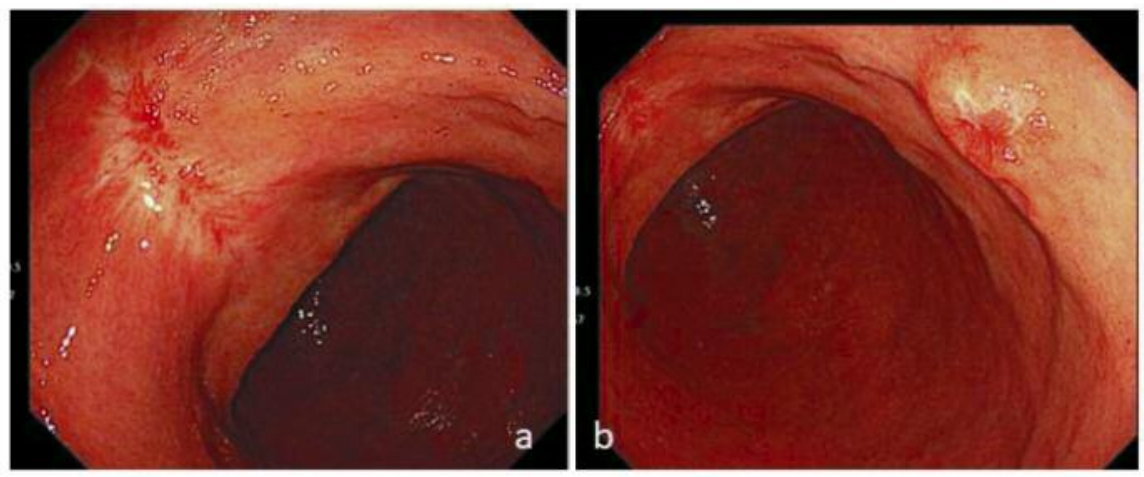

Figure 2. The endoscopic findings at 7 months after endoscopic resection. a) The irregularly changed scar was detected. b) The new low elevated lesion appeared at the posterior wall in the lower body of the stomach.
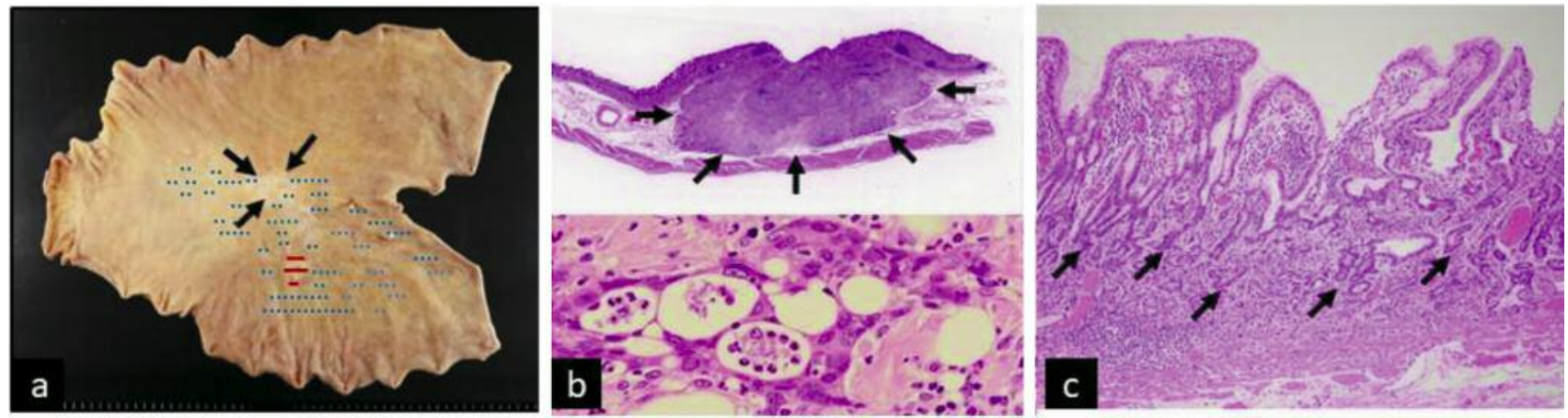

Figure 3. Macroscopic and pathological findings of the surgical and endoscopic specimen. a) The dotted blue lines represent the intramucosal cancer. The solid red lines represent the low elevated lesion at the posterior wall of the stomach. The arrows show the scar of endoscopic resection. b) The enlarged view of the low elevated lesion (solid lines) in Figure 3a. Poorly differentiated adenocarcinoma was detected in the submucosa. Top; low-power field. Bottom; high-power field. c) Histology of the dotted lines in Figure 3a. Irregularly fused glands were spread laterally in the lamina propria.

this type of GC is often ill-defined with indistinct borders due to the fact that the tumor grands called "crawl" cannot be detected in the superficial layer but in the epithelial proliferative zone (4). The irregularly fused glands show the characteristic features, but about $50 \%$ of the first biopsies are incorrectly interpreted as either indeterminate for neoplasia or reactive intestinal metaplasia because of the extremely low cellular atypia $(3,7)$. Therefore, the pathologists should diagnose this type of GC based on the structural atypia. These characteristics demonstrate a discrepancy between endoscopic and pathological examinations, which lead to the misdiagnosis or the high rate of positive lateral resection margins after endoscopic resection (6).

As described above, the endoscopic detection of "crawling type" GC is difficult. It is known that EWDA are frequently found in the middle-third of the stomach $(4,6)$. The tumor is superficial depressed or superficial flat type, and the margin is often indistinct. Usually, a submucosal infiltrative lesion cannot be observed. Kang KJ et al. reported that the rate of the positive resection margin for this type of gastric cancer is up to about $30 \%$ even after careful observations (6). We routinely use chromoendoscopy, magnifying endoscopy, NBI technique and endoscopic ultrasound (EUS) at diagnosis (810). In our case, the tumor was a superficial depressed (0-IIc) type found in the middle-third of the stomach, but the borders were relatively well defined as shown by the indigocarmine staining and NBI. As a result of these examinations, we regarded the tumor as endoscopically resectable. Though the endoscopic diagnostic ability is advancing, the observation of the lesion deeper than the gastric glands remains difficult. The endoscopic advances such as chromoendoscopy with an acetic acid-indigocarmine mixture or the further development 
of EUS might contribute to a more accurate diagnosis of this type of gastric cancer (11).

In the histological examinations, the cellular atypia is hard to detect, and the pathologists should diagnose it by the structural atypia. This type is often misdiagnosed as benign lesions such as intestinal metaplasia (12). It is reported that irregularly fused glands are the most important diagnostic clue for "crawling type" gastric cancer (1). The pattern of the fused glands with architectural features such as branching, anastomosing, distention, abortive and spiky forms, glandular overgrowth and discohesive neoplastic cells recreate the shapes of the letters "H", $\mathrm{X}$ ", "W" and "Y" $(3,7)$. As the irregularly fused gland often spreads sparsely and the structural atypia is difficult to diagnose in a small specimen, a sufficient amount of tissue would be necessary to distinguish from other lesions. So, the endoscopists should take biopsies from multiple sites around the suspicious area. In the cases which are difficult to diagnose, re-endoscopic observation or a re-biopsy should be considered. It has also been reported that the degenerative glands and the neoplastic glands are observed in the deeper sites of the mucosa (1). Thus, biopsies should be taken from all mucosal tissue layers, not only the superficial mucous tissue, in order to detect the irregularly fused glands in the deeper sites. In our case we retrospectively reviewed the ESD specimen and found such glands scattered in the lamina propria (Data not shown).

In the surgical specimen after distal gastrectomy, the glands were detected all over the GC lesions including the ESD lesion and the low elevated lesion. The grade of cellular atypia was very low compared to the structural atypia. As shown in Figure 3b, the poorly differentiated adenocarcinoma was detected in the submucosa only at the low elevated lesion, though the other part of the lesion showed very well differentiated adenocarcinoma.

In conclusion we reported a case of "crawling type" GC which was very difficult to diagnose preoperatively, but could be detected by an additional gastrectomy. This type of GC is still a challenge for endoscopic and histological diagnosis due to the few clues in the early stage of the disease. We should keep this variant in mind, especially when we observe superficial depressed or superficial flat type tumor in the middle of the stomach. Careful observation with multiple biopsies of all mucosal layer and a re-biopsy is the key procedure for obtaining the right diagnosis.

\section{References}

1 Okamoto N, Kawachi H, Yoshida T, Kitagaki K, Sekine M, Kojima K, Kawano T and Eishi Y: "Crawling-type" adenocarcinoma of the stomach: a distinct entity preceding poorly differentiated adenocarcinoma. Gastric Cancer 16: 220232, 2013.
2 Kushima R, Vieth M, Borchard F, Stolte M, Mukaisho K and Hattori T: Gastric-type well-Differentiated adenocarcinoma and pyloric gland adenoma of the stomach. Gastric Cancer 9: 177184, 2006.

3 Endoh $\mathrm{Y}$ and Watanabe $\mathrm{H}$ : Intestinal-type adenocarcinoma in the fundic gland area of the stomach. Stomach Intest 28: 1009-1023, 1994.

4 Ushiku T, Arnason T, Ban S, Hishima T, Shimizu M, Fukayama $\mathrm{M}$ and Lauwers Y: Very well-differentiated gastric carcinoma of intestinal type: analysis of diagnostic criteria. Modern Pathol 26: 1620-1631, 2013.

5 Niimi C, Goto H, Ohmiya N, Niwa Y, Hayakawa T, Nagasaka T and Nakashima N: Usefulness of p53 and Ki-67 immunohistochemical analysis for preoperative diagnosis of extremely welldifferentiated gastric adenocarcinoma. Am J Clin Pathol 118: 683-692, 2012.

6 Kang K, Kim K, Rhee P, Min B, Rhee J, Kushima R and Lauwers G: Gastric extremely well-differentiated intestinal-type adenocarcinoma: a challenging lesion to achieve complete endoscopic resection. Endoscopy 44(10): 949-952, 2012.

7 Koike M, Takizawa T and Iwasaki Y: Pathological aspect of early gastric carcinoma, handling of the endoscopically mucosectomized specimen and problem in the pathological diagnosis. Stomach Intest 28: 127-138, 1993.

8 Nagahama T, Yao K, Maki S, Yasaka M, Takaki Y, Matsui T, Tanabe H, Iwashita A and Ota A: Usefulness of magnifying endoscopy with narrow-band imaging for determining the horizontal extent of early gastric cancer when there is an unclear margin by chromoendoscopy. Am S Gastrointest Endosc 74: 1259-1267, 2011.

9 Sakai Y, Eto R, Kasanuki J, Kondo F, Kato K and Arai M: Chromoendoscopy with indigocarmine dye added to acetic acid in the diagnosis of gastric neoplasia: a prospective comparative study. Gastrointest Endosc 68: 635-641, 2008.

10 Lee B, Kim G, Park D, Kim DH, Jeon T, Park S, You H, Ryu D, Kim DU and Song G: Acetic acid-indigocarmine chromoendoscopy for delineating early gastric cancer: its usefulness according to histological type. BMC Gastroenterol 10: 97, 2010.

11 Kono Y, Takenaka R, Kawahara Y, Okada H, Hori K, Kawano S, Yamasaki Y, Takemoto K, Miyake T, Fujiki S and Yamamoto $\mathrm{K}$ : Chromoendoscopy of gastric adenoma using an acetic acid indigocarmina mixture. World J Gastroenterol 20: 5092-5097, 2014.

12 Joo M and Han S: Gastric-Type Extremely Well-Differentiated Adenocarcinoma of the Stomach: A Challenge for Preoperative Diagnosis. J Pathol Transl Med 50(1): 71-74, 2016. 